\title{
Scaling Column Imprints using Advanced Vectorization
}

\author{
Lefteris Sidirourgos ${ }^{*}$ \\ Systems Group \\ Dept. of Computer Science \\ ETH Zurich, Switzerland \\ lsidir@inf.ethz.ch
}

\author{
Hannes Mühleisen \\ Database Architectures Group \\ Centrum Wiskunde \& Informatica \\ Amsterdam, The Netherlands \\ hannes@cwi.nl
}

\begin{abstract}
Column Imprints is a pre-filtering secondary index for answering range queries. The main feature of imprints is that they are lightweight and are based on compressed bit-vectors, one per cacheline, that quickly determine if the values in that cacheline satisfy the predicates of a query. The main overhead of the imprints implementation is the many sequential value comparisons against the boundaries of a virtual equi-height histogram. Similarly, during query scans, many sequential value comparisons are performed to identify false positives. In this paper, we speed-up the process of imprints creation and querying by using advanced vectorization techniques. We also experimentally explore the benefits of stretching imprints to larger bit-vector sizes and blocks of data, using 256-bit SIMD registers. Our findings are very promising for both imprints and for future index design research that would employ advanced vectorization techniques and larger (up to 512-bit) and more (from 16 now to 32) SIMD registers.
\end{abstract}

\section{CCS CONCEPTS}

\section{-Information systems $\rightarrow$ Database query processing; Data} scans;

ACM Reference format:

Lefteris Sidirourgos and Hannes Mühleisen. 2017. Scaling Column Imprints using Advanced Vectorization. In Proceedings of DaMoN'17, Chicago, $I L$, USA, May 15, 2017, 8 pages.

DOI: http://dx.doi.org/10.1145/3076113.3076120

\section{INTRODUCTION}

Column Imprints [7] is a secondary index for answering range queries in a read optimized columnar database. Imprints are prefiltering bit-vectors that quickly determine if a cache line or block of data contains values that satisfy the range predicates of a query. They have been designed such that they are easy to build, typically as a side effect of the first range-scan query, and then subsequently used by all other queries. The imprints index structure is simple and lightweight, never exceeding $12 \%$ of the original size of the column, while speeding up significantly query execution times. Column Imprints are particularly useful for those attributes that

"this work was done while the author was at CWI.

Permission to make digital or hard copies of all or part of this work for personal or classroom use is granted without fee provided that copies are not made or distributed for profit or commercial advantage and that copies bear this notice and the full citation on the first page. Copyrights for components of this work owned by others than ACM must be honored. Abstracting with credit is permitted. To copy otherwise, or republish, to post on servers or to redistribute to lists, requires prior specific permission and/or a fee. Request permissions from permissions@acm.org.

DaMoN'17, Chicago, IL, USA

(c) 2017 ACM. 978-1-4503-5025-9/17/05 ..\$15.00

DOI: http://dx.doi.org/10.1145/3076113.3076120 are on the tail of a relational table, not worth the investment of building a primary (sort/cluster) index, yet often part of the many range predicates of a query.

Column Imprints are so efficient because they are built during a single sequential scan, where each value is compared against a number of boundaries of an equi-height histogram, in order to set the corresponding bit on a small bit-vector. An imprint is typically only 64-bits and stored as an unsigned long. Similarly, during query time, one sequential scan is needed over the imprints to quickly determine which blocks of data qualify for query evaluation. Even with such short description of the creation and usage of imprints, it is easy to assume that a vectorized programming framework with native CPU support will greatly benefit the performance of the index.

In this paper we make use of Intel's Advanced Vector Extensions [5] to speed up Column Imprints. SIMD instructions are used i) to efficiently compare multiple values against histogram boundaries, ii) to perform multiple bit-wise operations over imprints that extend beyond the standard 64-bit unsigned long words, and iii) to filter out false positive values at query time. These three points are the main computation intensive parts of the creation and query process of the index, and are exactly the ones that should be optimized by vectorization. As we will describe in detail in the next section, Column Imprints also employ compression techniques and dictionary-style bookkeeping, but these parts of the code are less often invoked and have many control-flow branching making them unsuited for SIMD optimization.

The scalar design of imprints is constrained by two important factors. First, the size of the imprint per cache line can not exceed 64-bits in order to achieve word alignment for the CPU registers. A larger imprint will break the bit-wise operations into more than one registers and thus the process will become significant slower. Second, each imprint encodes the values that fit in one cacheline, which typically is 64 bytes. The choice of one 64-bit imprint per cacheline is optimal, because it allows for a cache conscious implementation that avoids loading entire cachelines into L1 CPU cache memory if the pre-filtering stage determines not to. A data block larger than a cacheline will perform worse because of $i$ ) higher false positive ratios since it will set more bits in the limited 64-bit vector, ii) less than optimal data loading/streaming in the CPU cache, and iii) more cacheline lookup misses.

The aforementioned limitations can be easily overcome with the use of SIMD registers that extend beyond the 64-bit limit, to 128-bits, or 256-bits, or even (in the very near future) to 512-bits. With bigger imprint bit-vector sizes, more values can be encoded and thus bigger than a cacheline data blocks can be fetched with SIMD stream loading. In addition, loading data into multiple SIMD registers (16 registers currently, but soon to be increased to 32 ) 
allows for value comparisons in higher rates and bigger data blocks. In this work we investigate this potential by extending imprints up to 256-bits, and data blocks to 256 bytes.

The Instruction Set Extensions Programming Reference [5] states that "Intel AVX is designed to support 512 [...] bits in the future.". In accordance to that statement, the newer 2016 version of the manual [1] describes the new instruction sets for AVX-512 and lists future CPUs that will support it, including the Xeon Phi 2 which is already available. The AVX-512 instruction set has been long awaited, and many research in database engines design concludes with future work on 512-bit long registers [2]. We also anticipate this new hardware, and we are planning to extend imprints to use 512-bit vectors and 512 byte blocks per imprint.

Apart from the designing the SIMD version of the scalar implementation of Column Imprints, we also explore the research question of how good imprints scale with larger bit-vectors and data blocks. Given that we only have at our disposal a CPU with the AVX2 256-bit instruction set, we performed extensive experiments up to the 256-bit mark, and used our findings to project in the near future of the AVX-512 instruction set.

To recap, in this paper we make the following contributions:

- We investigate how modern wide vectorized instructions can improve performance of lightweight indexing structures such as Column Imprints.

- We present a SIMD enabled re-implementation of the scalar code of Column Imprints based on Intel's Advanced Vector Extensions (AVX2). The implementation is available as Open Source through a GitHub repository ${ }^{1}$.

- We perform an extensive experimental evaluation of our implementation on a modern processor and project our finding to the upcoming AVX-512.

We conclude our work with few thoughts on how we should design native SIMD indexes as opposed to adapting existing indexes to a vectorized version. A new line of research might be in sight, where vectorization is not an added benefit on top of a scalar implementation, but a design choice of the index itself. Extending this thought to other hardware accelerators, such as FPGAs, instead of trying to integrate them as a side component, we should aim for a seamless native support inside the database engine.

The remainder of this paper is structured as follows. In Section 2 we give an overview of the main design concepts behind the Column Imprints index. We continue with explaining the vectorized version of imprint construction and querying (Section 3). Then, Section 4 presents experimental results of a prototype implementation on thousands of data columns. Finally, Section 6 discusses results, research outlook, and future work.

\section{COLUMN IMPRINTS}

A Column Imprints index is a cache conscious secondary pre-filtering structure suitable for both low and high cardinality columns. The main purpose is to quickly identify which blocks of data do not store values that satisfy a range predicate, and thus prevent those blocks from being fetched into the CPU cache for further value comparisons. The index consist of three main components, a collection of small bit-vectors, called imprints, a dictionary that aligns

\footnotetext{
${ }^{1}$ https://github.com/lsidir/imprints
}

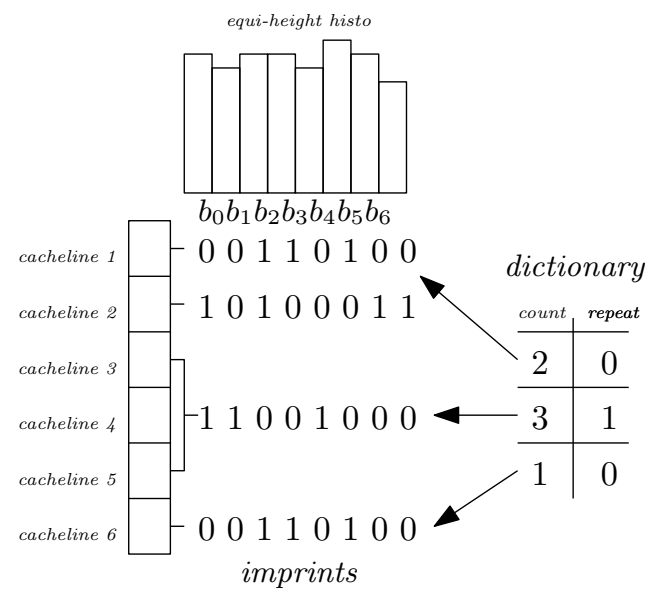

Figure 1: A Column Imprint Index

compressed imprints with the corresponding data blocks, and an array of boundaries that partitions the value space of the indexed column into equi-height bins (i.e., an equi-height histogram of the values distribution).

Figure 1 gives an overview of the index structures. Given a column with values from domain $\mathrm{D}$, an imprint index is constructed by first taking a small sample to approximate a histogram of a few (typically 64 or less) equal-height bins. These bins are used to derive the boundaries $b_{i}$ to be used to mark the range each bit in the imprint covers (top part of Figure 1). The entire column is then scanned, and for every cacheline of data, a bit-vector is created. The bits in each bit-vector correspond to the bins of the histogram. A bit is set if at least one value in the cacheline falls into the corresponding bin. The resulting bit-vector is an imprint of the current cacheline that describes which buckets of the approximated histogram the values of the cacheline fall into. As shown in Figure 1, an imprint does not have only one bit set per position, but as many bits as are needed to map all distinct values of a cacheline. The collection of all the resulting imprints form a unique Column Imprint. Consequently, by examining the imprints of a column, the execution engine can decide - in a cacheline granularity - which parts of the column data are relevant to the query predicates, and only then fetch them for further processing. Contrary to previous work, a column imprint is a non-dense bit indexing scheme, i.e., only one bit is set for all equal values in a cacheline, instead of the traditional approaches of bitmaps where each data point is always mapped to a different bit.

To reduce the memory footprint of imprints, a simple but powerful compression scheme is used. Consecutive and identical imprints are compressed together and annotated with a counter. The right side of Figure 1 shows a small dictionary example. The count column counts how many consecutive cachelines have unique imprints (i.e., one imprint per one cacheline), or how many consecutive cachelines share the same imprint (i.e., one imprint per many cachelines). The repeat column marks the one-to-one relationship between cachelines and imprints (repeat $=0$ ), or the many-to-one (repeat $=1$ ). This compression exploits the empirical observation that data suitable for secondary indexing exhibits, in the cacheline level, some degree of clustering or partial ordering. Column imprints are designed 

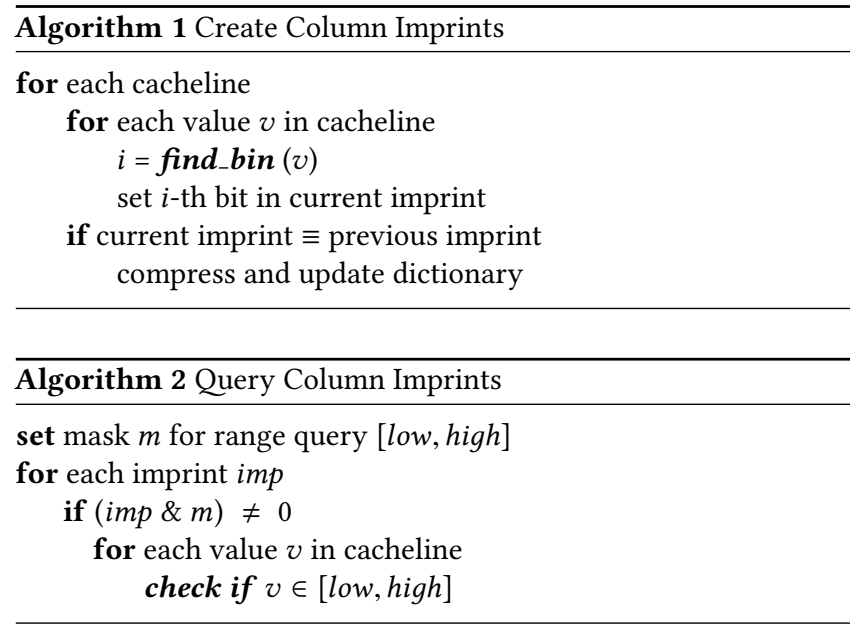

such that any clustering or partial ordering is naturally exploited without the need for extra parameterization.

Algorithms 1 and 2 provide a high level overview of the process to create and query imprints. The creation process is a single scan over the data, where for all values in a cacheline the function find $_{-}$bin is invoked to determine which bit in the imprint has to be set. This function call ${ }^{2}$ is the time-dominant operation for Algorithm 1. Function find_bin performs 64 comparisons of the form $v>b_{i}$ and it is exactly the part of the code that will be vectorized in the next section.

Similarly, for the query Algorithm 2, the process starts by scanning each imprint $i$ and comparing it with a mask bit-vector $m$. Mask $m$ has all bits that fall between the query range [low, high] set, i.e., $m[i]=1$ if low $<b_{i}<$ high. If imprint imp and mask $m$ have common bits set, then the cacheline has to be examined further for qualifying values and for rejecting false positives. This operation, denoted as check if in Algorithm 2 is the most time consuming part of querying, and it will be the subject of speeding up through vectorization in the next section.

For a complete presentation of Column Imprints and a detailed explanation of each algorithm we refer the reader to [7].

\section{VECTORIZED COLUMN IMPRINTS}

For completeness of the presentation in this paper, we include the time dominant code snippets of the scalar implementation of the imprints algorithms, as identified in the previous section and described in details in [7]. This work is about substituting these critical snippets of code in the imprints creation and querying algorithms in order to enable advanced vectorization optimizations.

\subsection{Imprints Creation}

The performance-critical part of imprints creation is the histogram bin assignment for each value from the column data, i.e., the code for find_bin function. Especially for wide imprints, it seems intuitive to implement a non-recursive binary search in the histogram boundary array $b[]$ to determine the correct bin $i$, such that $v>b_{i}$ and $v<b_{i+1}$. However, the large amount of branching required

\footnotetext{
${ }^{2}$ implemented as a macro to avoid function call overhead costs
}

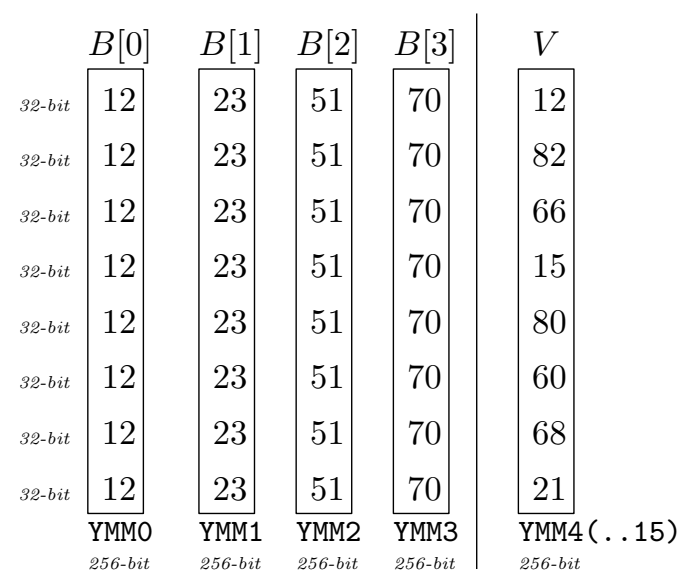

Figure 2: SIMD registers for imprints creation

makes this solution slower than a "brute-force" approach, where all boundaries $b_{i}$ are checked against the input value, regardless the outcome, and all comparison results are summed up. The result is the bin index of the respective value $v$. The following pseudo-code illustrates this approach:

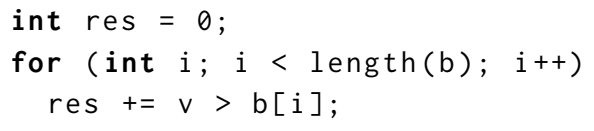

Vectorization of this approach is straightforward, depending on the input value type width $(8,16,32$ or 64 bits), we can ask the CPU to perform many (up to 64 for AVX-512) value-to-bin boundaries comparisons in a single SIMD instruction. This works because AVX comparison operators, for example_mm256_cmpgt_epi32, will return a vector where each element is set to -1 for each of the comparisons that evaluate to "true". These result vectors can then be summed up to yield the bin boundary index.

To illustrate this method, consider the following example. We assume 256-bit SIMD instructions, 32-bit integer input values, 4-bit imprints, and 8 values per imprint. The histogram boundaries $b_{i}$ in this example are $b_{0}=12, b_{1}=23, b_{2}=51$ and $b_{3}=70$. In a preparatory step, we compute SIMD vectors for all boundaries where all vector entries are set to the boundary value $b_{1}$ and store them in an array of vectors, denoted with $B$. We use uppercase variable names for SIMD vectors. This was found to be faster than creating the boundary vector $B$ ad-hoc, at the expense of some additional memory use. Figure 2 shows the four boundaries $b_{i}$ stored in the four SIMD registers $B[0]$ to $B[3]$, and the fifth SIMD register $V$ that contains the 8 values of the data block (cacheline) to be compared. The values in $V$ will be compared simultaneously, using SIMD instructions, with one boundary value at a time. Therefore, the vectorized bin boundary index computation proceeds as follows in four steps (SIMD comparisons): 


\begin{tabular}{l|l|rrrrrrrr} 
& $R E S$ & 0 & 0 & 0 & 0 & 0 & 0 & 0 & 0 \\
& $V$ & 13 & 82 & 66 & 15 & 80 & 60 & 68 & 21 \\
\hline 1 & $R E S+=(V>B[0])$ & -1 & -1 & -1 & -1 & -1 & -1 & -1 & -1 \\
2 & $R E S+=(V>B[1])$ & -1 & -2 & -2 & -1 & -2 & -2 & -2 & -1 \\
3 & $R E S+=(V>B[2])$ & -1 & -3 & -3 & -1 & -3 & -3 & -3 & -1 \\
4 & $R E S+=(V>B[3])$ & -1 & -4 & -3 & -1 & -4 & -3 & -3 & -1 \\
\hline & $R E S=0-R E S$ & 1 & 4 & 3 & 1 & 4 & 3 & 3 & 1
\end{tabular}

The subsequent step is to extract the individual entries from $R E S$ and look up the bit pattern for that particular histogram bin. All retrieved bit patterns are OR-ed together, creating the final imprint for a particular data block. In this example, the four-bit imprint would be 1101, since no entries fall into the second histogram bin. Furthermore, the resulting imprint needs to be checked against the imprint of the previous block of input values. This post-processing is not particularly performance-critical and we only use SIMD instructions for imprints that are larger than 64 bits, as we will explain later in this section in more details. This example is also simplified, usually more than eight values would be represented by an imprint. In this case, the imprint is the logical OR of the results of several runs of the described method.

Note that the bin boundaries for eight input values are determined using only 9 SIMD instructions. Using the sequential method described above, 64 individual comparison instructions would have been required to achieve the same result. As vector width increases over time, this ratio increases further. However, we have found that the performance of the bin index computation can be further increased by comparing the values against two (and not more) histogram boundaries in each iteration. The following code snippet shows this optimization. For each iteration along the boundaries array $B, V$ is compared (cmpgt) with two boundary vectors and the outcome is added together, and then added to the total result. We suspect this to be due to the lack of a data dependency within the first two steps of calculation in this version. Intel's Haswell, Broadwell, and Skylake architectures can execute at most two 256-bit SIMD instructions per cycle, which suggests a two-stage pipeline for those instructions. Hence, by removing the data dependency, we can get at most two instructions completed per cycle. Analysis of performance counters confirmed that the code here reached this maximum. As we will see in the experimental results, the speedup of roughly $15 x$ over the scalar code can probably be traced to this effect and implementation.

The following code snippet shows this optimization for 32-bit integer input values.

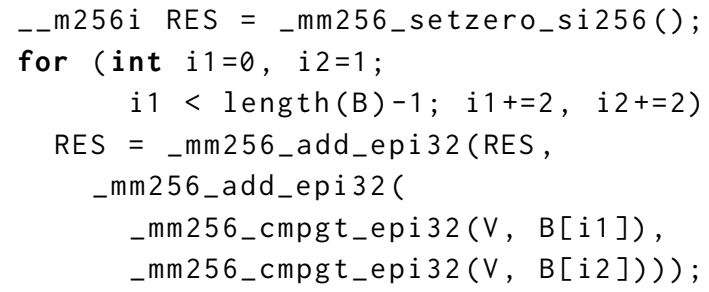

One limitation of this approach is that the number of histogram bin boundaries (and hence imprint length) is limited by the type of the input values. This is because a vectorized comparison operators returns comparison results of the same size in turn. If $V$ is of type char, then we use _mm256_cmpgt_epi 8 to compare the values to

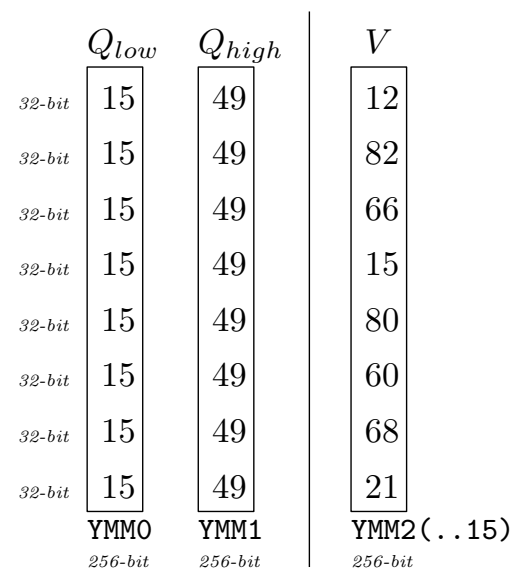

Figure 3: SIMD registers for imprints querying

boundaries, hence we can address at most 127 when we accumulate comparison results in RES. As a result, we cannot create 256-bit imprints for 8-bit values. This is not a big issue since it does not make a lot of sense to create 256 bins for at most 256 distinct values, unless we are aiming at a one-to-one bitmap indexing structure.

\subsection{Imprints Querying}

Querying the vectorized imprints is very similar to the scalar version with two exceptions. The comparison of both outer and inner range boundary bit masks with the imprint entries uses vectorized instructions instead of simple bitwise logic operations. This is done to support larger than 64-bit imprints. There are three possible outcomes of this comparison. First, the data values represented by the imprint has no overlap with the query range. In this case, the querying process simply advances to the next imprint. Second, there might be a match with only the bits of the mask that are inclusively entirely inside the range query, in which case all values represented by the imprint satisfy the query predicate without further checking. The interesting third case is when there is a partial overlap with the mask, which means that individual data values need to be compared with the range boundaries in order to determine which values satisfy the range query predicates. Here, SIMD operations are used to compare multiple values with the upper and lower query range boundaries. This comparison can be done in one instruction.

Consider the same value array $V$ as in the example of the previous section. Also consider the range query $Q=[15,49]$. The mask of this range query will be 0110 since $Q_{\text {low }}=15$ is larger than $b_{0}=12$ and $Q_{h i g h}=49$ is smaller than $b_{3}=70$. The mask 0110 has common bits set with the imprint of $V$ which was 1101, thus the values of $V$ have to be examined one by one. Figure 3 shows the two SIMD registers that store the $Q_{l o w}$ and $Q_{\text {high }}$ predicates of the range query. Similarly, the next SIMD register holds the values of $V$ that will be compared with $Q_{l o w}$ and $Q_{h i g h}$ in one go, using vectorization.

The following code snippet shows the SIMD version of comparing imprints with the query mask Q_MASK and checking the values 
against the low Q_LOW and high Q_HIGH ends of the query range for false positives.

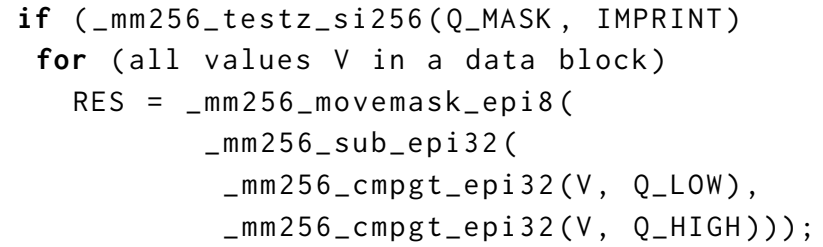

The RES variable has set the bits that correspond to the values of $V$ that satisfy the comparison with the low and high of the range query. It is straightforward afterwards to identify the qualifying values from the RES bit pattern.

\subsection{Larger Imprints}

Lastly, in order to support imprints larger than a 64-bit long word, we changed the type of an imprint from unsigned long to a _-mm256i. Therefore, the bitwise operations had to be substituted with SIMD instruction calls, such as_mm256_or_si256 for OR, and _mm256_xor_si256 for XOR.

Probably more interesting is the following code to check if two imprints have exactly the same bits set.

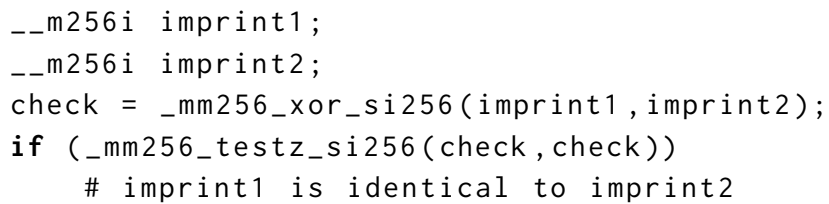

The function _mm256_testz_si256 will return true if all bits in check are 0 , but in order for this to happen imprint 1 and imprint 2 have to have all bits set exactly the same. Similarly, _mm256_testz_si256 function is used during the query process to check if an imprint has common bits set with the query mask, where the mask is also an _.mm256i type. We refer the reader to our code repository ${ }^{1}$ for further details about our implementation.

The changes presented in this section, from the scalar code to supporting SIMD instructions, accounts for a speedup up to 16 times. In the next section we evaluate these changes, and examine the benefits of using more histogram bins, i.e., wider imprints, together with larger than a cacheline data blocks.

\section{EXPERIMENTS}

To evaluate the performance of the vectorized version of imprints, we used a subset of the collection of datasets as in the original paper of Column Imprints [7]. The data sets consist of 6,476 different columns, with a maximum number of records of 600 millions, and contain integer and decimal types of various length. For a detailed overview of the data sets we refer the reader to [7]. The dataset used for experiments is available on request.

We created a stand-alone implementation of SIMD imprints, which is available for download ${ }^{1}$. We compared our SIMD-enabled version of imprints with the original scalar implementation of imprints.

We are interested in mainly investigating the impact of two parameters. First, the bit width of the imprint (number of bins). Note that wider imprints require more comparisons during index

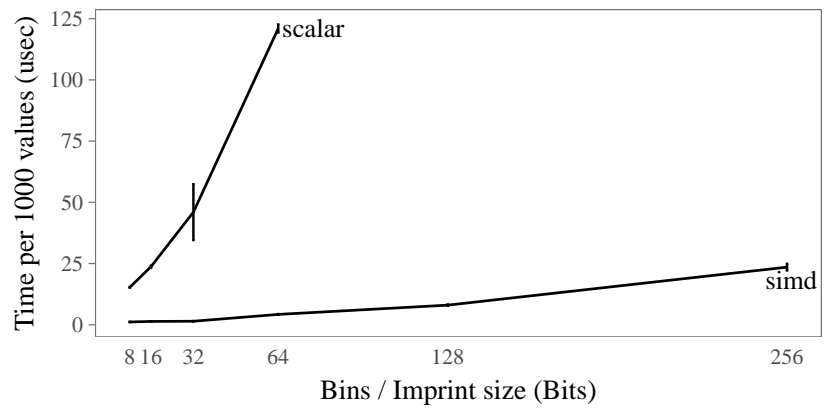

Figure 4: Imprint creation time vs. width.

creation, thus we expected to have a slowdown as the number of bins increase, but always to be many times faster than the equivalent scalar version. The benefit of using larger bit-vectors for imprints is that the false positive ratio is reduced, thus having to check less values during query time. The second parameter we investigate is the number of encoded values per imprint, or in other words, the size of the block of data. This parameter will have an influence over the precision of the imprints. More values per block can lead to a smaller index size, but can lead to a negative impact on query performance, as more individual values need to be checked for false positives. In our experiments, we vary the imprint size between 8 and 256 bits and the input block size (number of input values times their individual length) between 64 and 256 bytes. Other aspects of the imprints, such as the size and the compression percentage does not change with the SIMD-enabled version, so we do not repeat these experiments. Note that the compression percentage has a fixed upper limit, and it is always the ratio between the size of an imprint over the size of the data block. For each imprint configuration on each data column, we evaluate ten queries with even-spaced selectivity between $0 \%$ and $100 \%$.

All experiments were run on an Intel Core i7-6770HQ ("Skylake$\mathrm{H}$ ”) CPU clocked at $2.60 \mathrm{GHz}$. The system contained $32 \mathrm{~GB}$ of main memory. We also ensured that the files read are in the page cache before imprint creation.

All plots below show the average imprint creation or query time per 1,000 values over all data sets (and queries). In addition, the standard error is indicated as error bars. For creation, "values" refers to input data values, for querying, it refers to imprint index entries. This is done to allow a fair comparison between data sets of different sizes and different characteristics. For example, since the imprints index collapses subsequent equal imprint entries using dictionary encoding, the data distribution has a direct impact on the scanning effort. In extreme cases (a single constant value for the entire column), a single imprint entry can represent billions of data values. Hence this normalization.

\subsection{Imprint Creation}

We expect that the imprint size has a direct impact on index creation time, since every bit that is added requires additional comparison operations. However, we also expect that the SIMD implementation described in this work will significantly outperform the optimized scalar implementation. In the experiments in this section, we have 


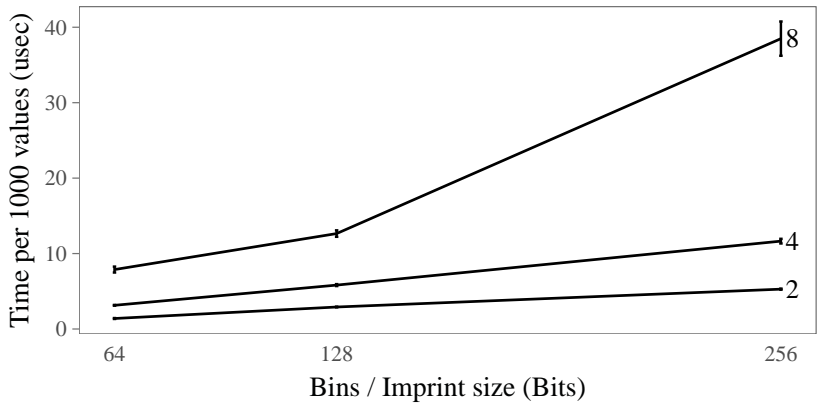

Figure 5: Imprint creation time for different value type widths (SIMD Only).

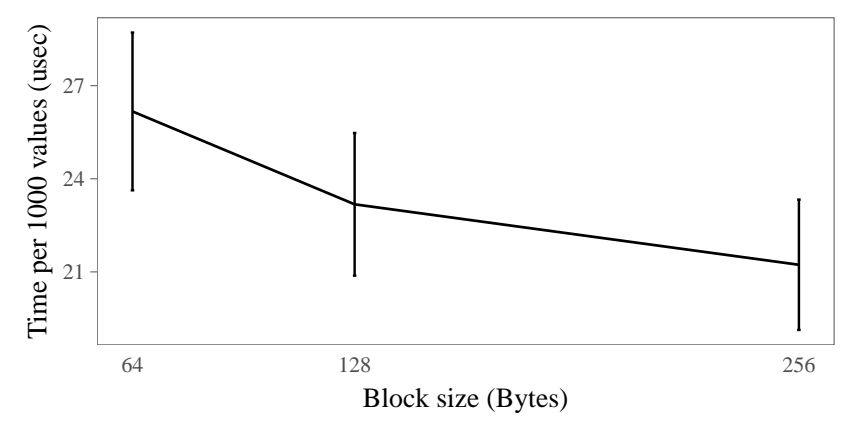

Figure 6: Imprint creation time for different block sizes encoded (SIMD Only, 256 Bins).

varied the imprint size for both implementations in order to study these expectations.

Figure 4 shows the outcome of this experiment. The scalar baseline and the SIMD implementation are shown as different lines. We can see how the time required to create imprints for 1,000 values scales linearly to the amount of bins and hence imprint size. We can also see how the SIMD version greatly outperforms the scalar version, with the largest possible imprint size of 256-bits taking about as much time as the scalar code for 16-bit imprints. For the 64-bit imprints, the scalar code required on average $120 \mu$ s per 1,000 values, while the SIMD implementation took only $4 \mu$ s with very low variance.

Drilling down, we further expect that the input type width has a significant impact on vectorized imprint creation time. For example, for input data values of type int16_t, 16 values can be boundarychecked in one SIMD instruction, while for int64_t values only 4 comparisons are possible in a single instruction. Figure 5 shows the imprint creation timing results (for the SIMD implementation only) by input data type width. As expected, we can see how data with 8 bytes input type width leads to the longest imprint creation time, while the 2 bytes data is fastest. For 256-bit imprints, the 2 bytes values took on average of $5.3 \mu$ s per 1,000 values, while the 8 bytes type width took $38.5 \mu \mathrm{s}$.

Turning towards the second parameter, the amount of input data values per imprint entry, we expect that fewer values per imprint

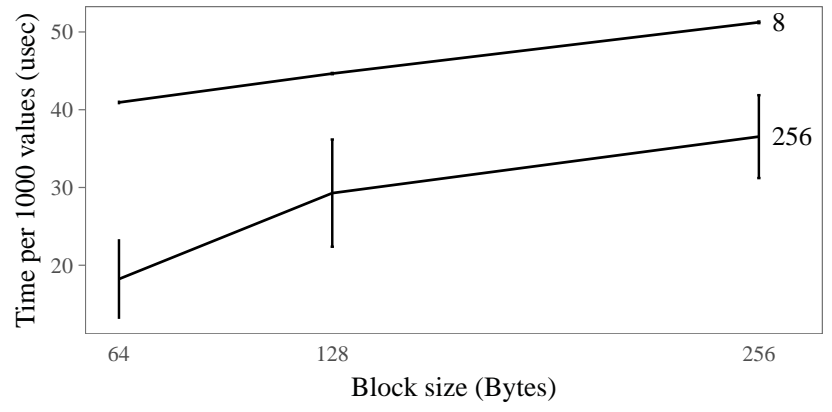

Figure 7: Imprint querying time for different block sizes (SIMD Only).

will improve creation time since fewer imprint candidates need to be created and compared with the previous entry for dictionary compression purposes. For this experiment, we have varied the number of input values per imprint between 8 and 128 in such a way that the input block size (the amount of input values multiplied by their type length) ranges between 64 and 256. Figure 6 shows the results of this experiment. We can see how indeed the imprint creation time drops significantly if more data is encoded into a single value. However, a larger block size will also lead to reduced precision, which has an adverse effect on query run time, which we will investigate next.

\subsection{Imprint Querying}

Querying performance is a trade-off between to extremes. On the one end, the imprint index is empty, requiring a full scan of the data values. On the other end, the imprint index is a one-to-one (bit-)mapping of the data. While both are technically valid, we are searching for a more balanced trade-off. This trade-off is controlled by imprint length and block size.

We expect that larger block sizes will decrease query performance (as more entropy is lost), but it is unclear by how much. Figure 7 plots the time required to process 1,000 imprint index entries against increasing block sizes. Two lines are shown, one for 8and one for 256-bits imprint length. We see that query performance indeed decreases as block size is increased, but (on average) at most linearly. It is very likely that query performance will degrade for even larger block sizes, certainly if data values have to be fetched from disk.

In the original imprints paper, a 8-to-1 relationship between data value bits and (before duplicate elimination) imprint bits was found to work best. When scaling this up to larger imprints, we expect this relationship to still hold. Figure 8 shows a rather complex behavior of the queries. However, the basic assumption that a 8-to1 relationship between data and index still holds. For the 64 bytes block size, we observe good performance for an imprint size of 64bits. For the 256 bytes blocks, 256-bits imprints size showed the best performance, which confirms our expectations. A similar result was found for the (not plotted) 128 byte block size with 128-bits imprints. 


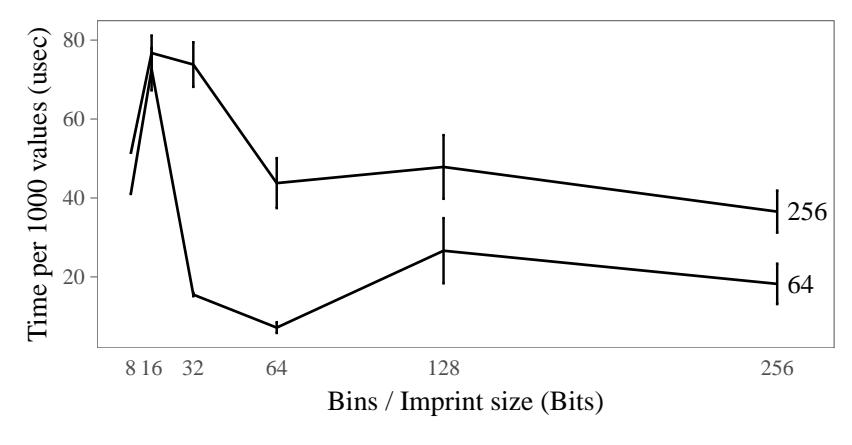

Figure 8: Imprint querying time for different amounts of values encoded (SIMD Only).

Overall, we argue that the experimental results show good scalability of imprint indexes thanks to the availability of vectorized instructions.

\section{RELATED WORK}

There has been a large amount of previous work related to using SIMD vectorization to speed up analytical data management tasks. Early papers demonstrated how existing implementations of relational operators could be sped up using SIMD instructions [9]. Generally speaking, systems that use columnar or (data-)vectorized storage models can benefit from a straightforward translation from scalar code implementing relational operators to the equivalent vectorized code.

A more thorough operator redesign was shown to be required to to fully take advantage of vectorized instructions [6]. The authors used selective load and store and scatter/gather operations available in modern SIMD instruction sets as building blocks for new scan and join operators. Experimental results show that for example for low selectivity, vectorized code can provide an approximately 10 times throughput improvement in scans. Overall, the authors found that vectorization favors cache-conscious algorithms and that the speedup provided by vectorization is independent of other optimizations.

A paper comparing sort and hash join algorithms [2] already reported the observation that sort-based join algorithms scale nearlinearly with the SIMD width. The paper also predicted that sortbased join algorithms are expected to show better performance than hash-based approaches with a SIMD width of 512-bits or higher. This shows the relevance of the increased vector widths that are now becoming available.

Data layout adaption is a third option apart from the previously discussed operator re-implementation and algorithmic redesign. One paper proposes to adapt in-memory data layout in such a way that it is amenable to SIMD processing [4]. In such layout, every SIMD word contains bits from a large amount of data values, which allows early pruning of data blocks in selections based on prefix comparisons or improved look-up performance.

The overall research progression in transforming scalar code to vectorized could be described by the following chart with representative references.

\author{
Operator Re-Implementation with SIMD Instructions \\ $\downarrow$ \\ SIMD-Aware Algorithm Design \\ $\downarrow$ \\ SIMD-Aware Data Layout Adaption
}

\section{CONCLUSION \& RESEARCH OUTLOOK}

In this paper we have demonstrated that by substituting the expensive scalar code snippets of column imprints with the equivalent single-instruction multiple-data code snippets, we can achieve a speed up of almost 16 times. The exercise of finding the equivalent SIMD version is an interesting one, and requires some work to identify the correct code snippets to be changed. SIMD instructions benefit from continues sequential loading, while control flow branching has to be avoided always. These observations makes the job more challenging, and can lead to different index design choices. For example, we plan to investigate the possibility of dropping altogether the compression features of Column Imprints (which require some if-else statement) in favor of non-interrupting sequential loads (i.e., memory streaming) and bulk bit-wise comparisons.

looking for false positives, thus splitting the process of uninterrupted bulk operations and conditional branching.

Another important aspect of our work is the expansion from 64-bit word registers to the equivalent 256-bit SIMD registers, and therefore the increase of the imprints width. The, soon to come, AVX-512 which will support 32 512-bit SIMD registers will allow for even more performance boost for imprints.

However, the interesting observation is that not only Column Imprints but other bit-vector based techniques can benefit from SIMD instructions. We believe that there is interesting work to be done here, and we intend to extend this work to other techniques such as WAH bitmap compression [8].

Another promising direction to speed up querying is to precompute all possible result offset vectors similar to [3]. For example, if the values $(1,5,6,3)$ are checked for the range query [2, 5], only the second and last entry are true positives. For this case, the pre-computed vector (NULL, 0, NULL, 1) can be looked up, and then add the base index for this block of values, say 42 , using vectorized instructions, thus we have efficiently created the output candidate list (NULL, 42, NULL, 43). AVX has a feature to control which elements from a SIMD register should be copied into contiguous main memory, making final assembly of the result efficient as well.

A final thought on research outlook is that, although now we are successfully trying to adjust existing index structures to the SIMD era, we should start designing new indexes that have native support for vectorization. Complex compression, multiple branching, and other structures that aimed at loading less data in the CPU might be abandoned for the use of SIMD instructions such as_mm256_stream_load_si256 that allow stream loading with non-temporal memory hints. The benefits of such bulk loads may overweight the benefits of less data transfer through control flow statements.

\section{ACKNOWLEDGMENTS}

Stefan Manegold of the CWI Database Architectures Group optimized the scalar imprints code. This work was partially funded 
by the Netherlands Organisation for Scientific Research (NWO), project "Capturing the Laws of Data Nature" (Mühleisen).

\section{REFERENCES}

[1] 2016. Intel Architecture Instruction Set Extensions Programming Reference. https://software.intel.com/sites/default/files/managed/c5/15/ architecture-instruction-set-extensions-programming-reference.pdf

[2] Changkyu Kim, Eric Sedlar, Jatin Chhugani, Tim Kaldewey, Anthony D. Nguyen, Andrea Di Blas, Victor W. Lee, Nadathur Satish, and Pradeep Dubey. 2009. Sort vs. Hash Revisited: Fast Join Implementation on Modern Multi-Core CPUs. PVLDB 2, 2 (2009), 1378-1389. http://www.vldb.org/pvldb/2/vldb09-257.pdf

[3] Harald Lang, Tobias Mühlbauer, Florian Funke, Peter A. Boncz, Thomas Neumann, and Alfons Kemper. 2016. Data Blocks: Hybrid OLTP and OLAP on Compressed Storage using both Vectorization and Compilation. In Proceedings of the 2016 International Conference on Management of Data, SIGMOD Conference 2016, San Francisco, CA, USA, fune 26 - July 01, 2016. 311-326. DOI : http://dx.doi.org/10.1145/2882903.2882925

[4] Yinan Li and Jignesh M. Patel. 2013. BitWeaving: Fast Scans for Main Memory Data Processing. In Proceedings of the 2013 ACM SIGMOD International Conference on Management of Data (SIGMOD '13). 289-300. DOI : http://dx.doi.org/10.1145/ 2463676.2465322

[5] Chris Lomont. 2011. Introduction to Intel Advanced Vector Extensions. https: //software.intel.com/sites/default/files/m/d/4/1/d/8/Intro_to_Intel_AVX.pdf

[6] Orestis Polychroniou, Arun Raghavan, and Kenneth A. Ross. 2015. Rethinking SIMD Vectorization for In-Memory Databases. In Proceedings of the 2015 ACM SIGMOD International Conference on Management of Data (SIGMOD '15). 14931508. DOI : http://dx.doi.org/10.1145/2723372.2747645

[7] Lefteris Sidirourgos and Martin L. Kersten. 2013. Column imprints: a secondary index structure. In Proceedings of the ACM SIGMOD International Conference on Management of Data, SIGMOD 2013, New York, NY, USA, fune 22-27, 2013. 893-904. DOI : http://dx.doi.org/10.1145/2463676.2465306

[8] Kesheng Wu, Ekow J. Otoo, and Arie Shoshani. 2006. Optimizing Bitmap Indices with Efficient Compression. ACM Trans. Database Syst. 31, 1 (March 2006), 1-38. DOI : http://dx.doi.org/10.1145/1132863.1132864

[9] Jingren Zhou and Kenneth A. Ross. 2002. Implementing database operations using SIMD instructions. In Proceedings of the 2002 ACM SIGMOD International Conference on Management of Data, Madison, Wisconsin, Fune 3-6, 2002. 145-156. DOI : http://dx.doi.org/10.1145/564691.564709 